\title{
La maladie de Wilson: le gène en cause est similaire au gène responsable de la maladie de Menkes
}

Dans les numéros de décembre 1993 de Nature Genetics [1-3] et de Biochemical and Biophysical Research Communications [4], trois groupes ont rapporté l'identification du gène responsable de la maladie de Wilson. Comme cela avait été prédit $(\mathrm{m} / \mathrm{s}$, $n^{\circ}$ 3, vol. 9, p. 316), il s'agit d'un gène qui code pour un nouveau transporteur de cuivre appartenant à la famille des ATPases de type P, similaire au gène responsable de la maladie de Menkes [5-7].

Le cuivre est considéré comme un oligo-élément indispensable à toute forme de vie. En effet, des enzymes telles que la cytochrome c oxydase, la superoxyde dismutase, la tyrosinase, la dopamine $\beta$ hydroxylase, la lysyl oxydase utilisent les ions $\mathrm{Cu}^{2+}$ comme co-facteurs. En excès, le cuivre est aussi un ion très toxique, capable d'oxyder les protéines et les lipides membranaires, de se lier aux protéines et aux acides nucléiques et, enfin, de favoriser la production de radicaux libres. Les différents organismes vivants doivent donc être dotés de mécanismes efficaces et adéquats leur permettant d'assurer l'homéostasie et le transport de ce métal lourd essentiel et de lutter contre les effets toxiques d'un éventuel excès des ions cuivre.

Actuellement, on connaît au moins deux maladies héréditaires dues à des anomalies du métabolisme du cuivre: la maladie de Menkes et sa forme allélique moins sévère, le cutis laxa lié à l'X, et la maladie de Wilson. La maladie de Menkes (Tableau I) [8] est caractérisée par une dysmorphie, une dégénérescence neurologique associée à un retard mental, un état particulier des cheveux qui sont dépigmentés et crêpelés, un déficit des parois vasculaires et une évolution rapide vers une mort précoce (en général avant l'âge de 5 ans). La majorité de ces signes cliniques pourrait être expliquée par les déficits en enzymes dont l'activité nécessite les ions cuivre. L'hypothèse, proposée depuis plusieurs années, selon laquelle le défaut primaire affecterait une protéine impliquée dans le transport intracellulaire du cuivre [8,9], a été étayée par l'identification du gène responsable de la maladie de Menkes, rapportée par Vulpe et al. [5], Chelly et al. [6], Mercer et al. [7]. Ces derniers travaux ont révélé que le produit du gène de Menkes serait un transporteur de cuivre, membre de la famille des ATPases de type P, dont le domaine $\mathrm{N}$-terminal, formé de six répétitions de 23 acides aminés contenant chacune le motif GlyMet-Thr-Cys-X-X-Cys (X étant n'importe quel acide aminé), est vraisemblablement impliqué dans la liaison du cuivre $\left(\mathrm{m} / \mathrm{s}, n^{\circ} 3\right.$, vol. 9, p. 316). Une localisation de la protéine du gène de Menkes au niveau du réticulum endoplasmique a été suggérée par les études immunocytochimiques rapportées récemment par J. Gitlin (résultats non publiés). Cette protéine serait donc impliquée dans la livraison du cuivre aux protéines nouvellement synthétisées.

La première description clinique de la maladie de Wilson (dégénérescence hépato-lenticulaire autosomique récessive, Tableau I) [8] a été rapportée en 1912 par KinnearWilson. Les corrélations entre les effets toxiques de l'accumulation du cuivre dans le foie et le cerveau et les signes cliniques de la maladie ont été décrites durant les années
1940. Cependant, malgré une compréhension limitée des mécanismes de transport et du métabolisme du cuivre, un traitement efficace utilisant des agents chélateurs comme la pénicillamine a été introduit par Walshe en 1956. Bien que le déficit primaire n'ait pas été connu, des études biochimiques avaient suggéré que la pathogénie de la maladie de Wilson serait caractérisée par deux perturbations fondamentales du métabolisme du cuivre: une réduction du taux d'incorporation du cuivre au niveau de la céruloplasmine, associée à une réduction de l'excrétion biliaire du cuivre. Ce dernier défaut serait responsable de l'accumulation du cuivre dans le foie et de la dégénérescence hépatolenticulaire progressive. Ultérieurement, le dépôt du cuivre dans le cerveau et la cornée entraîne respectivement une perte de la coordination des mouvements et la formation des anneaux de KayserFleischer. Par ailleurs, l'accumulation du cuivre dans d'autres tissus pourrait entraîner des troubles variés tels une perturbation tubulaire rénale, une ostéoporose, une arthropathie, une cardiomyopathie ou un hypoparathyroïdisme.

L’identification du gène responsable de la maladie de Menkes et son expression dans tous les tissus sauf le foie, dans lequel le niveau d'expression est très réduit, avaient suggéré que la maladie de Wilson était due, elle aussi, au défaut d'un transporteur du cuivre, spécifique du foie dans ce cas. Utilisant des approches similaires de clonage positionnel, cette hypothèse a été récemment confirmée de manière indépendante par les groupes de 


\begin{tabular}{|c|c|c|}
\hline \multicolumn{3}{|c|}{ Tableau I } \\
\hline & Maladie de Menkes & Maladie de Wilson \\
\hline Localisation & Xq13.3/récessive & 13q14.3/récessive \\
\hline $\begin{array}{l}\text { Données } \\
\text { cliniques }\end{array}$ & $\begin{array}{l}\text { Début à la naissance } \\
\text { Dysmorphie, retard mental } \\
\text { Dégénérescence neurologique } \\
\text { Cheveux dépigmentés et crêpelés } \\
\text { Déficit des parois vasculaires } \\
\text { Evvolution grave et rapide } \\
\text { Mort précoce } \\
\text { (en général avant } 5 \text { ans) }\end{array}$ & $\begin{array}{l}\text { Début durant l'enfance } \\
\text { Dégénérescence hépatolenticulaire } \\
\text { progressive } \\
\text { Mouvements involontaires } \\
\text { Ostéoporose, arthropathie } \\
\text { Anneaux de Kayser-Fleischer } \\
\text { Atteinte rénale } \\
\text { Cardiomyopathie }\end{array}$ \\
\hline $\begin{array}{l}\text { Données } \\
\text { biologiques }\end{array}$ & $\begin{array}{l}\text { Baisse de la cuprémie } \\
\text { Baisse du taux de la céruloplasmine } \\
\text { plasmatique } \\
\text { Baisse du taux du } \mathrm{Cu} \text { dans le foie }\end{array}$ & $\begin{array}{l}\text { Augmentation de la cuprémie } \\
\text { Augmentation du taux de la céruloplasmine } \\
\text { plasmatique } \\
\text { Augmentation de la cupréurie } \\
\text { Accumulation du Cu dans le foie }\end{array}$ \\
\hline $\begin{array}{l}\text { Culture } \\
\text { cellulaire }\end{array}$ & $\begin{array}{l}\text { Accumulation intracellulaire du } \mathrm{Cu} \text { par } \\
\text { défaut de relargage }\end{array}$ & Normal dans la majorité des cas \\
\hline Pathogénie & Défaut d'absorption intestinale $\mathrm{du} \mathrm{Cu}$ & $\begin{array}{l}\text { Diminution de l'excrétion biliaire et de } \\
\text { l'incorporation dans la sidéroplasmine } \\
\text { du Cu }\end{array}$ \\
\hline Traitement & Pas de traitement efficace & $\begin{array}{l}\text { Agents chélateurs: pénicillamine } \\
\text { et sels de zinc }\end{array}$ \\
\hline $\begin{array}{l}\text { Modèles } \\
\text { animaux }\end{array}$ & Mottled mouse $(\mathrm{mo})$ & $\begin{array}{l}\text { toxic milk mouse }(\mathrm{t} x) \\
\text { Chien terrier Bedlington }\end{array}$ \\
\hline $\begin{array}{l}\text { Produit } \\
\text { du gène }\end{array}$ & $\begin{array}{l}\text { ATPase de type } P \\
\text { Transporteur de cuivre }\end{array}$ & $\begin{array}{l}\text { ATPase de type } P \\
\text { Transporteur de cuivre }(60 \% \text { d'identité avec } \\
\text { le gène Menkes) }\end{array}$ \\
\hline Expression & Tous les tissus sauf le foie & Foie, rein et placenta \\
\hline Mutation & $16 \%$ de délétions & $\begin{array}{l}\text { Mutations ponctuelles } \\
\text { Petites délétions }\end{array}$ \\
\hline
\end{tabular}

Cox (Toronto, Canada) [1], Tanzi (Boston, MA, USA) et Gilliam (New York, NY, USA) [2, 3], et Gitlin, (Saint-Louis, MO, USA) [4], dont le père avait décrit en 1952 le déficit en céruloplasmine associé à la maladie de Wilson [10].

Des études de génétiques de liaison publiées en 1985 avaient montré que le locus de la maladie de Wilson ségrégeait avec une enzyme érythrocytaire, l'estérase D, localisée sur le chromosome 13[11]. Ulté- rieurement, le locus fut assigné à une région génomique flanquée par les marqueurs d'ADN proximal D13S31 et distal D13S59. L'établissement d'une carte physique et le clonage de la région d'intérêt, utilisant des banques génomiques spécifiques du chromosome 13, avaient permis l'isolement de marqueurs microsatellites physiquement ordonnés, et conduit à l'identification d'haplotypes spécifiques de la maladie de Wilson [12]. Afin d'isoler
l'ADNc du gène responsable de la maladie de Wilson, Bull et al. [1] utilisèrent comme sonde un fragment d'ADNc du gène de Menkes correspondant au domaine potentiel de liaison du cuivre. Les clones de YAC et de cosmides spécifiques du chromosome 13 contenant des séquences homologues à ce domaine furent identifiés après hybridation à faible stringence. Par la suite, des expériences de sélection d'ADNc permirent d'isoler des clo- 
nes d'ADNc localisés dans la région correspondant au locus de la maladie de Wilson et dont la séquence était similaire à celle du gène de Menkes. Tanzi et al. [2] se servirent d'un oligonucléotide dégénéré, correspondant à une séquence de liaison des métaux lourds, décrite dans le précurseur de la protéine $\beta$ amyloïde [12], pour identifier un clone d'ADNc de cerveau produit par la région génomique d'intérêt. Yamaguchi et al. [4], enfin, employèrent aussi l'hybridation à faible stringence pour isoler un clone d'ADNc de foie similaire à la partie $5^{\prime}$ du gène de Menkes.

L'ARNm, d'environ $7,5 \mathrm{~kb}$, produit par le gène responsable de la maladie de Wilson est exprimé essentiellement dans le foie, le rein $[1,4]$ et le placenta [2]. Le transcrit a été aussi détecté, mais à un niveau beaucoup plus faible, dans le cœur, le cerveau, le poumon, le muscle et le pancréas. L'étude du gène et de son transcrit chez les patients a révélé cinq mutations spécifiques de la maladie : deux transversions, une délétion et une insertion d'une paire de nucléotides $[2,3]$, et une délétion de sept paires de nucléotides [1].

La séquence nucléotidique de l'ADNc, obtenue par Cox et al., a permis de prédire que le transcrit code pour une protéine de 1411 acides aminés. Il s'agit d'un nouveau transporteur de cations, membre de la famille des ATPases de type $P$, très similaire au produit du gène de Menkes $[1,2]$. Le niveau global d'identité entre les produits des gènes de Wilson et de Menkes est de l'ordre de $56 \%$, mais un niveau supérieur d'identité a été observé dans les domaines de déphosphorylation $(78 \%)$, transduction-phosphorylation $(89 \%)$, liaison de l'ATP $(79 \%)\left(m / s, n^{\circ} 3\right.$, vol. 9, p. 316). Quant au domaine Nterminal, qui contient les six motifs potentiels de liaison du cuivre, son niveau d'identité entre les produits des deux gènes est de l'ordre de $65 \%$. Par ailleurs, l'analyse de la séquence peptidique du gène de Wilson a révélé aussi des différences de structure potentielles, telles que la présence d'une courte séquence hydrophobe proche du quatrième motif de liaison du cuivre.

L'ensemble des données rapportées dans les références [1-8] montre donc que les gènes responsables des maladies de Menkes et de Wilson codent pour des transporteurs de cations appartenant à la même famille, vraisemblablement impliqués dans le transport du cuivre. Bien que très significatives, ces données ne permettent pas encore de comprendre de manière satisfaisante les relations entre les déficits primaires et la physiopathologie de chacune de ces deux maladies. Apparemment, l'absence d'un gène de Menkes fonctionnel entraîne essentiellement un défaut d'absorption intestinale du cuivre, associé, d'une part à l'accumulation intracellulaire de cet élément, sous sa forme non toxique, dans tous les tissus de l'organisme, sauf le foie, et, d'autre part, à un déficit en enzymes qui utilisent les ions $\mathrm{Cu}^{2+}$ comme co-facteurs. Au contraire, l'absence de gène de Wilson fonctionnel entraîne la réduction de l'excrétion biliaire du cuivre et de l'incorporation de ce métal dans la céruloplasmine, associée à une accumulation du cuivre, sous sa forme toxique, dans plusieurs tissus essentiels. Bien que les données préliminaires concernant les profils d'expression des deux gènes et la localisation de la protéine du gène de Menkes puissent clarifier certaines questions, seules des études fonctionnelles détaillées, immunocytologiques et toxicologiques, pourront nous éclairer sur les mécanismes de transport du cuivre, non seulement intracellulaire, mais aussi entre les différents tissus. L'exploration de modèles animaux, tels que la souris mottled (mutation mo) équivalente à la maladie de Menkes, la souris toxic milk (mutation tx), le chien terrier Bedlington et le rat LEC, équivalents à la maladie de Wilson, devrait aider à élucider les mécanismes impliqués dans le métabolisme du cuivre et fournir des modèles adéquats pour le développement de nouvelles thérapeutiques.
Parmi les ATPases de type $P$ des eucaryotes, seuls les produits des gènes responsables des maladies de Menkes et de Wilson sont connus pour leur implication dans le transport des métaux lourds. Les protéines ATPases de type $P$ possèdent toutes un résidu aspartate qui est transitoirement phosphorylé à partir d'ATP durant le cycle de transport du cation, d'où le nom ATPase de type P. Les protéines codées par les gènes de Menkes et de Wilson partagent une analogie de structure avec plusieurs opérons de résistance aux métaux lourds tels que la CadA ATPase de Staphylococcus aureus et la $\mathrm{Cu}^{2+}$ ATPase Cop B d'Enterococcus hirae, non seulement dans les domaines communs à toutes les protéines ATPases de type $\mathrm{P}$, mais aussi dans le domaine dithiol $\mathrm{N}$-terminal qui est supposé contenir les motifs de liaison des métaux lourds [14]. La conservation de ces ATPases transporteurs de métaux lourds chez des organismes aussi divergents que les bactéries fournit des possibilités intéressantes pour l'étude fonctionnelle des différentes régions des protéines codées par les gènes de Menkes et de Wilson

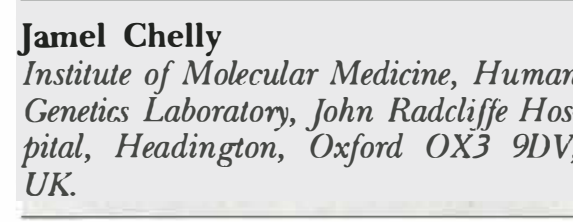

\section{RÉFÉRENCES}

1. Bull PC, Thomas GR, Rommens JM, Forbes JR, Cox DW. The Wilson disease gene is a putative copper transporting Ptype ATPase similar to the Menkes gene. Nature Genet 1993; 5 : 327-37.

2. Tanzi RE, Petrukhin K, Chernov I, el al. The Wilson disease gene is a copper transporting ATPase with homology to the Menkes disease gene. Nature Genel 1993; 5 : 344-50. 
3. Petrukhin K, Fischer SG, Pirastu M, el al. Mapping, cloning and genetic characterization of the Wilson disease gene. Nalure (ienel $1993 ; 5$ : 338-43.

4. Yamaguchi Y, Heiny ME, Gitlin JD. Isolation and charaterization of human liver cDNA as a candidate gene for the Wilson disease. Biochem Biophys Res Commun 1993; $197: 271-7$

5. Vulpe C, Levinson B, Whitney S, Packman S, (itschier J. Isolation of a candidate gene for Menkes disease and evidence that it encodes a copper-transporting ATPase. Nature (jenet 1993; 3: 7-13.

6. Chelly J, Tümer Z, Tфnnesen T, el al. Isolation of a candidate gene for Menkes disease that encodes a potential heavy metal binding protein. Nalure Genet 1993; 3: 14-9.

7. Mercer LFB, Livingston J, Hall B, Paynter JA, Begy C, Chandrasekharappa S, Lockhart P, Grimes A, Bhave M, Siemieniak D, Glover TW. Isolation of a partial candidate gene for Menkes disease by positional cloning. Nature (ienet 1993; $3: 20-5$.

8. Dankes DM. In : Beaudet AL, Sly W'S Valle 1), eds. Metabolic basis of inherited disease. New York: Mc(iraw-Hill, 1989: 1411-31.

9. Herd SM, Camakaris J, Christofferson R, Wookey P, Danks DM. Uptake and efflux of copper-64 in Menkes'-disease and normal continuous lymphoid cell lines. Biochem / 1987 ; 247 : 341-7.

10. Scheinberg IH, Gitlin D. Deficiency of ceruloplasmin in patients with hepatolenticular degeneration (Wilson's disease). Srience 1952; 116: 484-5.

11. Frydman M, Bonné-Tamir B, Farrer LA, el al. Assignment of the gene for Wilson disease to chromosome 13 : linkage to the esterase D locus. Proc Nall Acad Sci LISA $1985 ; 82: 1819-21$.

12. Thomas GR, Roberts EA, Rosales T(), et al. Allelic association and linkage studies in Wilson disease. Hum Mol (jenel 1993; 2 : 1401-5.

13. Bush AI, Pettingell $W$, Wasco $W$, Tanzi RE. Zinc-induced precipitation of $\beta 4 A$ is prevented by copper. Soc Neurosi Abs 1993; 19: 19.

14. Silver S, Nucifora G, Phung LT. Human Menkes $X$ chromosome disease and the staphylococcal cadmium-resistance ATPase : a remarkable similarity in protein sequence. Mol Microbiol 1993; 10: 7-12.

\section{TIRES A PART}

J. Chelly. 